\title{
The 3D Solid Modeling Technology and Its Applications in Machine Design
}

\author{
Feng Guanglin ${ }^{1, a}$ \\ ${ }^{1}$ College of Mechanical and Electrical Engineering, Shunde Polytechnic, Foshan, 528300, China \\ afgl_0921@163.com (corresponding author)
}

Keywords: Machine design; 3D geometry modeling; 3D solid modeling technology; Modeling

\begin{abstract}
The 3D solid modeling technology is the basic of the virtual model in the virtual reality, as well as is the core element of the CAD system. It has quickly become the mainstream of theCAD technology. Computational geometry is to use the computer to transform, synthesize and analyze the physical geometry information. The 3D modeling technology can be divided into the following three types: (1) point-line modeling technology (2) plane modeling technology (3) 3D solid modeling technology.
\end{abstract}

\section{Introduction}

The 3D solid modeling technology is the basic of the virtual model in the virtual reality, as well as is the core element of the CAD system. It has quickly become the mainstream of the CAD technology. The 3D modeling methods in CAD consist of the point-line modeling technology, the plane modeling technology and the 3D solid modeling technology. Generally, we call the real 3D object volume pixel. There are two kinds of the volume pixel: (1) scanning volume pixel (2) basic volume pixel. The scanning volume pixel can make the 2D plane become the 3D solid model by stretching, rotating, etc. It can create many 3D solid model with complex shapes and high-speed. The basic volume pixel can only create some very basic entities, such as the cone, the rectangle, the cylinder, the sphere. Computer creates complex shapes to construct a solid model by storing basic volume pixel and Boolean operations.

\section{The Theory of the 3d Geometry Modeling Technology}

In 1970s, the engineer A.R.Forrest gave the definition of the computational geometry for the first time: computational geometry is to use the computer to transform, synthesize and analyze the physical geometry information. Physical geometry information can be divided into two categories: one is the topology information, it provides the structural relations and adjacency relations between the physical elements; and the other is the geometry information, it provides the geometric characteristics and the locations of the physical elements. The technology and theory of the 3D geometry modeling is the perfect combination of the computational geometry, computer science and the interactive planes display technology.

\section{The Types of 3D Modeling Technology}

The 3D modeling technology can be divided into the following three types:

(1) The point-line modeling technology

The point-line modeling technology began in the 60 s and 70s of the last century to solve the problem that how to figure the form in the internal computer of the automatically drawing. It focuses on the physical boundary lines. This technology in the application of the plane form is very efficient, however, there're many problems when involving the form with curved surface because that the boundaries of the form is not always straight lines, they changes with the observer viewpoint and the perspective and these contours can't be determined. Such problems must be solved by the plane 
modeling technology. The point and line of the point-line modeling technology, the plane and the independent body frame model are the mainstreams of that time 3D geometry modeling.

(2) The plane modeling technology

The plane model is to use the collection of the plane to represent objects and to use the ring to define the boundary of the surface on the basis of the point-line model increasing the information of the object surface. We call the part surrounded by the boundaries in the point-line model plane, it forms the plane model. The plane model involves the curved surface model. The curved surface modeling technology mainly studies the controlling, representation and analysis of the curved surface. It's based on the parametric surfaces. The plane model expands the application range of the point-line model to meet the light and dark color chart, line and surface blanking, the plane and plane intersection, CNC machining needs. The plane model is based on the polygonal meshes. It has some shortcomings, mainly in the model. We only get a surface, we don't know which side the object exists, it only represents the plane boundary without the true body properties, such as the moment of inertia, centroid and mass. The plane model can't calculate and analyze the overall properties of the object, nor can regard this object as a whole to examine the interrelated properties with other objects, such as the intersection. Plane model overcomes many shortcomings of the point-line model, completely defining the 3D plane. It can represent the non-analytic objects.

(3) The 3D solid modeling technology

The 3D solid modeling is to define which side the objects exist on the basis of the plane model. As a 3D representation, the 3D solid modeling technology usually consists of some basic elements such as wedge, rectangle and cylinder which we use their intersection, parallel and difference to represent the objectsl. The 3D solid modeling can calculate the mass, centroid, volume and moment of inertia as well shape. This technology doesn't use the process of point, line, plane and body to complete the mode as split entities, analyze and optimize the finite element mesh because it has a variety of information of the object. In the 1970s, the British IC.Braid proposed that using the wedge, cylinder and cube to create complex objects by intersection, parallel and difference. After that, the theory and technology of the 3D solid modeling develops rapidly. In fact, the definition of the 3D solid modeling has been proposed in the 1960s. Just because the theory isn't very mature, it isn't applied in the CAD/CAM system until 1970s. After 1980s, a number of practical 3D solid modeling systems have been introduced.

\section{The Modeling Method of 3D Solid Modeling Technology}

During the 3D modeling, we need many geometry parameters of the model. Firstly, we should establish the coordinate system, and then input the location of the points, the length of the lines, the size of the plane and the stretching direction of the body, on the basis of the above operations, we set the rotation, drawing, chamfer angle and the round angle. The following are the basic characteristics of the establishment.

(1) The stretching

A sketch profile, moving along the direction perpendicular to the sketch plane to create the entity is the tensile characteristics(see Fig.1).

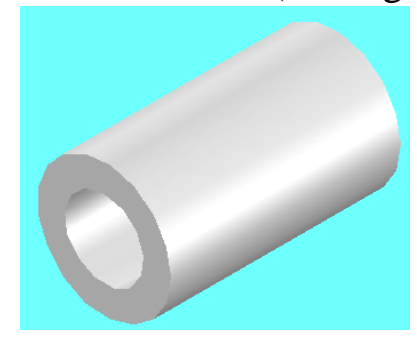

Fig. 1 The 3D stretching solid modeling

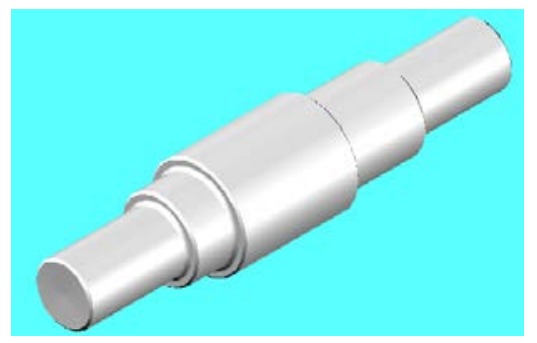

Fig.2 The 3D stretching modeling 
(2) The rotation

A sketch profile, rotating around one axis to create the entity is the rotational characteristics(see Fig.2).

(3) The scanning

A sketch profile, moving along the route curve to create the entity is the scanning characteristics(see Fig.3).

(4) The lofting

Smoothly connecting the multiple interface sketches to create the entity is the lofting characteristics.

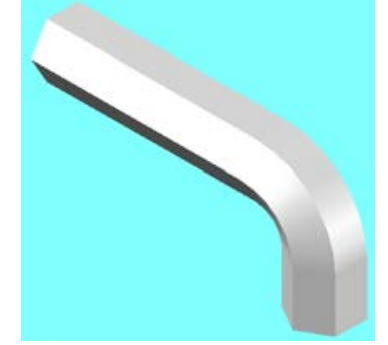

Fig.3 The 3D scaning solid modeling

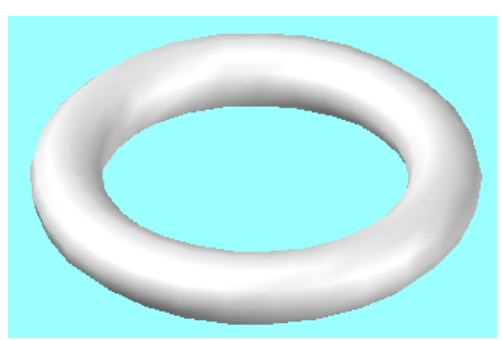

Fig.4 The 3D ring solid modeling

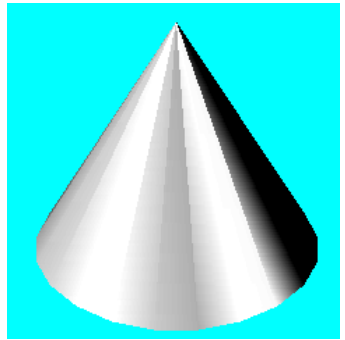

Fig.5 The 3D cone solid modeling

(5) The ring

After establishing the coordinate system of the ring model, we should input the following parameters: the radius of the ring, the base point coordinate, the initial angle and the final angle, the ring edge number.

(6) The cone

When establishing the cone model, we should input the following parameters: the height, the radius, the base point coordinate, rotating angle and edge number.

(7) The sphere

When modeling, we should carry out the 3D rotation transformation and the 3D shift transformation, move the vertices to the base point and calculate the vertices of the sphere using the origin of coordinate as the base point. We should input the following parameters: zoom factor, base point coordinate and radius.

(8) The rectangle

When modeling, we should input the following parameters: length, width, height, base point coordinate and the rotation angle.

(9) The cylinder

When modeling, we should input the following parameters: the up radius, the down radius, the base point coordinate, the height, the rotation angle and the edge number.

\section{The Application in The Machine Design of the 3d Solid Modeling Technology}

The 3D solid modeling and its intersection, parallel and difference operations provide the ideal development tool to generate the complex entities, it has important research and application value in the CAD field and it's a difficulty in the geometric modeling field.

For example, we can model a base using the 3D solid modeling technology. The base is composed of the rectangular units, round table, cylinder and other basic elements, and then we can carry out the solid operation to complete the base modeling. According to the elements' features, we establish the above elements in order and at last, we establish a base model by removal and Boolean operations (see Fig.6). 


\section{Conclusions}

When modeling, we begin with establishing the coordinate system, and input the points' position and the length of the lines, at last, set the rotation and stretching characteristics. Through the above steps, we can establish the basic 3D objects, such as the rectangle, the cylinder and the cone. We can even build a car model through advanced modeling. The application of modern machinery industry can't do without CAD, and the basic part of the CAD technology is the solid modeling, so studying the 3D solid modeling is very important to the modern engineers.

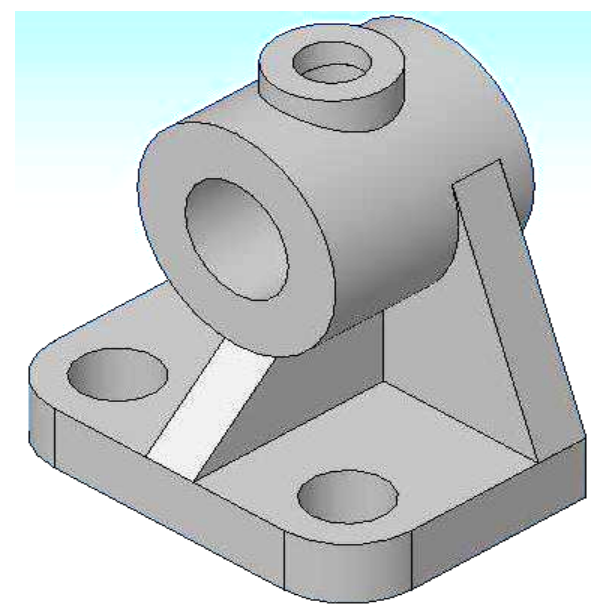

Fig.6 The 3D solid modeling technology of the base

\section{References}

[1] Reguicha AA, Voelker HB.Boolean Operations in Solid Modelling, Boundary Evaluation and Merging Algorithm[J]. Proceeding IEEE, 1985,73(l):18-25.

[2] Weiler K.Edge-based data structures for solid modeling in curved-surface environments[J]. IEEE Computer graphics and application, 1985,5(1):21-40.

[3] Mantyla M.Boolean Set Operations of 2-manifolds through Vertex Neighborhood Classification[J]. Transactions on Graphics, 1986,5(1):14-19.

[4] Li Weishi, Li Jiangxiong. A new judgment of the planar polygon direction and points[J]. Journal of Computer-Aided Design and Computer Graphics, 2000,12(6):405-407.

[5] Wu Jian, Zheng Kangping. A method to test whether the point exists in the polygon and the polyhedron[J]. Journal of Chinese Computer Systems, 2003,24(12):2200-2203.

[6] Changqing Gao, Shukun Cao, Wei Wang, etc, Disk Cam and Motion Simulation Based on SolidWorks, Journal of Modern Manufacturing Technology and Equipment, 2008(5): pp:60-62.

[7] Wei Lu, Lian Liu, Parametric Design and Motion Simulation of Cam Mechanism, Journal of Electrical Engineering Technology,2008(2):pp61-63. 